\title{
Polaronic and Nonadiabatic Phase Diagram from Anomalous Isotope Effects
}

\author{
P. Paci, ${ }^{1}$ M. Capone,${ }^{2}$ E. Cappelluti, ${ }^{2}$ S. Ciuchi,${ }^{3}$ C. Grimaldi, ${ }^{4}$ and L. Pietronero ${ }^{1}$ \\ ${ }^{1}$ Dipartimento di Fisica, Università di Roma "La Sapienza," and INFM UdR RM1, 00185 Roma, Italy \\ 2 "Enrico Fermi" Research Center, clo Compendio del Viminale, and INFM UdR RM1, 00184 Roma, Italy \\ ${ }^{3}$ Dipartimento di Fisica, Università de L'Aquila, and INFM UdR AQ, 67010 Coppito-L'Aquila, Italy \\ ${ }^{4}$ Ecole Polytechnique Fédérale de Lausanne, LPM Station 17, CH-1015 Lausanne, Switzerland
}

(Received 3 May 2004; published 25 January 2005)

\begin{abstract}
Isotope effects (IEs) are powerful tools to probe directly the dependence of many physical properties on lattice dynamics. In this Letter we investigate the onset of anomalous IEs in the spinless Holstein model by employing the dynamical mean field theory. We show that the isotope coefficients of the electron effective mass and of the dressed phonon frequency are sizable also far away from the polaronic crossover and mark the importance of nonadiabatic lattice fluctuations. We draw a nonadiabatic phase diagram in which we identify a novel crossover, not related to polaronic features, where the IEs attain their largest anomalies.
\end{abstract}

The relevant role of electron-phonon (e-ph) interactions on the properties of complex materials as the high- $T_{c}$ superconductors has been recently revived by a number of experiments. In particular, the finite, and yet unexplained, anomalous isotope effects (IEs) on the penetration depth [1], on the pseudogap temperature [2], and on the angle-resolved photoemission spectra [3] in high- $T_{c}$ cuprates open a new challenge in understanding the role of the $e$-ph interaction in these materials. Anomalous IEs appear also in the magnetic and charge-ordering critical temperatures of manganites, pointing out the relevance of the $e$-ph coupling also in these materials [4].

Despite the $e$-ph problem having been thoroughly studied, only a few partial studies have been devoted to IEs and to their significance in relation to the underlying nature of the $e$-ph interaction [5-7]. Yet, the prediction and observation of IEs on different physical properties represent a powerful tool to assess the role of the $e$-ph interaction in many materials. For instance, the finite isotope shift on the low temperature penetration depth [1], and hence indirectly on the charge carrier effective mass $m^{*}$, is of particular interest since it contrasts the conventional Migdal-Eliashberg (ME) scenario which predicts strictly zero IE on this quantity. In this perspective the understanding of finite IEs on $\mathrm{m}^{*}$ cannot rely on the ME framework, and more general approaches are then required.

A modern tool of investigation that overcomes the limitations of ME theory is the dynamical mean field theory (DMFT), a nonperturbative method which neglects spatial correlations in order to fully account for local quantum dynamics, and which becomes exact in the infinite coordination limit [8]. In the case of $e$-ph interactions, this approach allows us to study with equal accuracy all coupling regimes, and to fully include phonon quantum fluctuations which are only partially taken into account in the ME approach according to Migdal's theorem. DMFT has been successfully employed in the study of multiphonon effects, polaron instabilities, and metal-insulator transi- tions (MIT), as well as quasiparticle regimes of the Holstein electron-phonon Hamiltonian [9-12], which can be considered as a paradigmatic lattice model for the $e$-ph interaction.

In this Letter, we discuss the anomalies of the IEs on electronic and phononic properties arising in purely $e$-ph systems by employing DMFT to span the whole parameter space of the Holstein model, determined by the dimensionless $e$-ph coupling $\lambda$ and by $\gamma=\omega_{0} / t$, i.e., the "adiabatic" ratio between the phonon energy $\omega_{0}$ and the electron hopping rate $t$. We show that a sizable negative isotope coefficient on the electronic mass $m^{*}$ characterizes the whole parameter range, while a divergence of $\alpha_{m^{*}}$ is recovered only at the MIT polaron transition at $\lambda=\lambda_{c}$ and $\gamma=0$. Similar features are found for the renormalized phonon frequency $\Omega_{0}$ which displays an isotope coefficient $\alpha_{\Omega_{0}}$ significantly different from the ME limit $\alpha_{\Omega_{0}}=1 / 2$. Based on the dependence of the isotope coefficients on the adiabatic ratio $\gamma$, we draw a phase diagram wherein we identify, beside the strong-coupling polaronic regime [11]: (i) a nonadiabatic perturbative regime where IEs increase with $\gamma$, and (ii) a complex nonadiabatic regime where the anomalies of the IEs decrease with $\gamma$ and approach the Lang-Firsov predictions in the $\omega_{0} / t \rightarrow \infty$ limit.

In our study we are interested in the continuous evolution of the $e$-ph properties from the quasiparticle to the polaronic regime. It is well known that the Holstein model undergoes various instabilities leading to superconductivity, charge-density-wave ordering, and bipolaron formation [13]. In order to focus on the metallic properties and to clarify the origin of anomalous IEs in this regime, we consider here a half-filled spinless Holstein model, which enforces the metallic character in the whole $\lambda-\gamma$ space (for $\gamma \neq 0$ ) [11]. Our Hamiltonian reads

$$
H=-t \sum_{\langle i, j\rangle} c_{i}^{\dagger} c_{j}+g \sum_{i} n_{i}\left(a_{i}+a_{i}^{\dagger}\right)+\omega_{0} \sum_{i} a_{i}^{\dagger} a_{i},
$$


where $c_{i}^{\dagger}\left(c_{i}\right)$ and $a_{i}^{\dagger}\left(a_{i}\right)$ are creation (annihilation) operators for electrons and phonons on site $i$, respectively, $n_{i}=c_{i}^{\dagger} c_{i}$ is the electron density, and $g$ is the $e$-ph matrix element.

The physical properties of Eq. (1) are governed by two microscopic parameters: the $e$-ph coupling $\lambda=2 g^{2} / \omega_{0} t$ and the adiabatic ratio $\gamma=\omega_{0} / t$. In the adiabatic limit $\gamma=0$, the standard Landau Fermi-liquid (FL) picture is sustained by the ME theory for $\lambda<\lambda_{c}$, while for $\lambda>\lambda_{c}$ the FL regime is destroyed due to the polaron localization. For $\gamma>0$, this sharp transition becomes a smooth crossover separating well and poorly defined quasiparticle excitations. This phenomenology is reflected in the appearances of anomalous IEs on various quantities. Let us consider, for example, the isotope coefficient on the effective electron mass $m^{*}: \alpha_{m^{*}}=-d \ln \left(m^{*}\right) / d \ln (M)$, where $M$ is the ionic mass. Since $g \propto 1 / \sqrt{M \omega_{0}}$ and $\omega_{0} \propto$ $1 / \sqrt{M}$, the $e$-ph coupling $\lambda$ is independent of $M$ and $\alpha_{m^{*}}$ can be rewritten as

$$
\alpha_{m^{*}}=\frac{1}{2} \frac{d \ln \left(m^{*} / m\right)}{d \ln (\gamma)} .
$$

According to the FL description, $m^{*}$ can be expressed in terms of a mass-enhancement factor $f_{m^{*}}$ :

$$
m^{*} / m=1+f_{m^{*}}(\lambda, \gamma) .
$$

In the adiabatic regime $\gamma \ll 1, f_{m^{*}}$ can be expanded in powers of $\gamma, m^{*} / m \simeq 1+f_{m^{*}}(\lambda, 0)+\gamma f_{m^{*}}^{1}(\lambda)$, and hence

$$
\alpha_{m^{*}}=\frac{\gamma}{2} \frac{m}{m^{*}} f_{m^{*}}^{1}(\lambda)
$$

The isotope coefficient thus increases with $\gamma$, and it correctly reproduces the ME result $\alpha_{m^{*}}=0$ when $\gamma \rightarrow 0$. Such an increase is, indeed, found by calculations based on a perturbative expansion in $\gamma[5,6]$, whose validity is, however, limited only to weak $\lambda$ values. In the opposite antiadiabatic limit $\gamma \rightarrow \infty$ the Holstein-Lang-Firsov approximation gives $m^{*} / m \simeq \exp (\lambda / 2 \gamma)$, leading to

$$
\alpha_{m^{*}}=-\frac{\lambda}{4 \gamma}
$$

which decreases as $\gamma$ gets higher, as opposed to the previous case of Eq. (4). The two limiting cases for $\gamma \ll 1$ and $\gamma \gg 1$ suggest that for fixed $\lambda<\lambda_{c}$ the strongest isotope shifts lie in the intermediate nonadiabatic region $\gamma \lesssim 1$, which can be investigated only by nonperturbative tools as the DMFT approach.

In this work we consider Eq. (1) on an infinite coordination Bethe lattice and use exact diagonalization (ED) to solve the impurity problem that DMFT associates with the lattice model $[8,14]$. As usual, the Anderson model is truncated by considering $N_{s}$ impurity levels, and a cutoff on the phonon number is imposed on the infinite phonon Hilbert space. The DMFT self-consistency is implemented in the Matsubara frequencies $\omega_{n}=(2 n+1) \pi \tilde{T}$ where $\tilde{T}$ is a fictitious temperature. The evaluation of IEs is a particularly difficult task since it requires a high accuracy on both the electron and phonon properties and of their dependence on $\gamma$. In particular, a number of phonon states up to 100 and $\tilde{T} / t$ as small as $1 / 1600$ were needed to ensure reliable and robust results. The number of impurity levels has been fixed at $N_{s}=9$, having checked that no significant change occurred for larger $N_{s}$. We compute the electron selfenergy $\Sigma\left(\omega_{n}\right)$ and the phonon Green's function $D\left(\omega_{m}\right)$ which yield the effective electron mass $m^{*} / m=$ $1-\Sigma\left(\omega_{n=0}\right) / \pi \tilde{T}$ and the renormalized phonon frequency $\Omega_{0}$ as $\left(\Omega_{0} / \omega_{0}\right)^{2}=-2 D^{-1}\left(\omega_{m=0}\right) / \omega_{0}$. The corresponding isotope coefficients are obtained by means of a finite shift $\Delta \gamma / \gamma=0.15$.

In Fig. 1 we show $m^{*} / m$ and $\Omega_{0} / \omega_{0}$ and their corresponding isotope coefficients as a function of the $e$-ph coupling $\lambda$ for $\gamma=0.1,1$, and 10, which are representative, respectively, of the quasiadiabatic, nonadiabatic, and antiadiabatic regimes. The polaron crossover is reflected in a strong enhancement of $m^{*} / m$ as $\lambda$ increases. The crossover occurs at $\lambda=1.18$ for $\gamma=0$ [12], and moves to larger couplings as $\gamma$ increases, all the way up to the antiadiabatic regime, in which the crossover roughly occurs when the average number of phonons $\alpha^{2}=\lambda / 2 \gamma \gtrsim 1$ $[9,11,15]$. As a consequence, at fixed $\lambda$ the effective mass becomes smaller as $\gamma$ increases, implying a negative isotope coefficient $\alpha_{m^{*}}$ as reported in Fig. 1(b). The polaron regime is thus identified by the huge negative values of $\alpha_{m^{*}}$. Like $m^{*} / m$, increasing $\gamma$ smooths the dependence of $\alpha_{m^{*}}$ on $\lambda$.

A similar behavior is found for the renormalized phonon frequency $\Omega_{0}$, as shown in Fig. 1(c), where the polaron

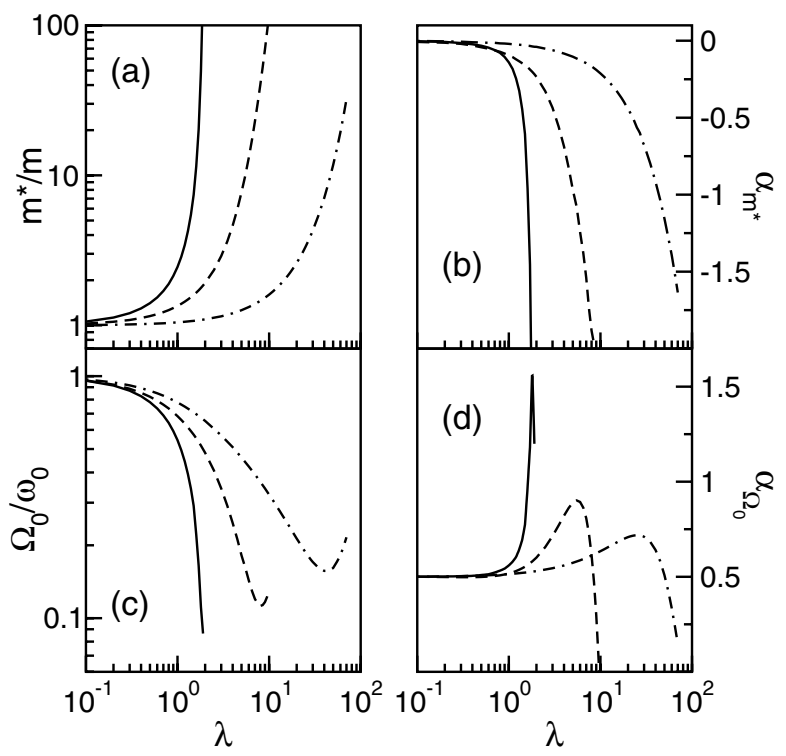

FIG. 1. (a) Effective electron mass $m^{*} / m$, and (c) renormalized phonon frequency $\Omega_{0} / \omega_{0}$ as a function of $\lambda$ for $\gamma=0.1$ (solid line), $\gamma=1$ (dashed line), and $\gamma=10$ (dotdashed line). (b),(d) The corresponding isotope coefficients. 
instability is reflected in a sharp phonon softening in the quasiadiabatic case $(\gamma=0.1)$ close to polaron crossover. Once again, the softening is weaker as $\gamma$ gets larger. For fixed $\lambda$ this leads to an anomalous isotope coefficient $\alpha_{\Omega_{0}}>1 / 2$, as reported in Fig. 1(d).

It is worth noting the apparent phonon hardening accompanied by the corresponding decreasing of $\alpha_{\Omega_{0}}$ as reported in Figs. 1(c) and 1(d) for large $\lambda$. This anomalous phonon feature is just a consequence of our "static" definition of renormalized phonon frequency $\left(\Omega_{0} / \omega_{0}\right)^{2}=$ $-2 D^{-1}\left(\omega_{m=0}\right) / \omega_{0}$. This definition corresponds to describe the full phonon spectrum as a single $\delta$ function at frequency $\quad \Omega_{0}^{2}=-1 /\left(\pi \omega_{0}\right) \int_{0}^{\infty} d \omega \operatorname{Im} D\left(\omega+i 0^{+}\right) / \omega$. Such a description becomes less representative as the spectral function acquires a complex structure.

In Fig. 2 we report the evolution of the phonon spectral function by increasing the $e$-ph coupling $\lambda$ for $\gamma=1$. The real frequency phonon propagator is directly computed in the ED scheme. Note that only gross features can be extracted because of the discreteness of the impurity model. The average phonon softening (thick red line) at small $\lambda$ stems from a transfer of spectral weight from $\omega \simeq$ $\omega_{0}$ to a low energy peak which exponentially approaches $\omega=0$. An opposite behavior occurs for strong $e$-ph coupling where the lattice potential is a double well with energy barrier $\Delta E \gg \omega_{0}$. This gives rise to a second peak at frequency $\omega_{0}$ yielding a hardening of the averaged phonon frequency $\Omega_{0}$. Similar considerations lead to the decrease at large $\lambda$ of the IE $\alpha_{\Omega_{0}}$.

The $\lambda$ dependence of $\mathrm{m}^{*} / \mathrm{m}$ and $\Omega_{0}$, with their respective IEs, highlights the appearance of giant IEs related to the polaronic crossover. However, sizable deviations of IEs from the ME predictions appear also far from the polaronic regime, in a region where the system preserves good metallic properties. Let us then analyze in more detail the dependence of the IEs on the quantum lattice fluctuations triggered by the finite $\gamma$. In Fig. 3 we plot the $\gamma$

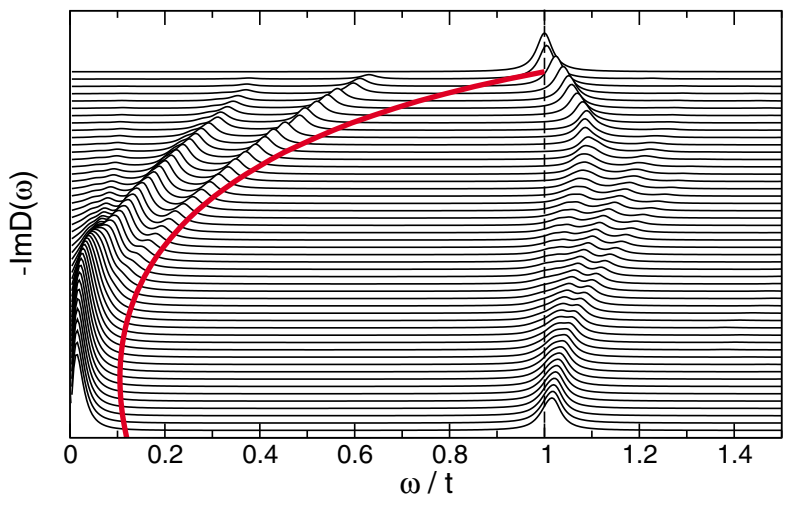

FIG. 2 (color online). Phonon spectral function (solid lines) for different values of the $e$-ph coupling $\lambda$ from 0 to 10 with steps of 0.2 . The thick line represents the renormalized phonon frequency $\Omega_{0}$ and the thin dashed line $\omega_{0}$. dependence of $\alpha_{m^{*}}$ and $\alpha_{\Omega_{0}}$ for different values of $\lambda$ ranging from weak $(\lambda=0.6)$ to intermediate-strong coupling $(\lambda=1.6)$. Two qualitatively different behaviors are identified. Dashed lines represent strong-coupling $\lambda$ values for which the system undergoes a polaronic MIT for $\gamma \rightarrow 0$ signaled by the divergence of the isotope coefficients. Solid lines are representative of weak-to-moderate $\lambda$ values for which the system maintains its FL metallic character with isotope coefficients recovering their standard ME values $\alpha_{m^{*}}=0$ and $\alpha_{\Omega_{0}}=1 / 2$ as $\gamma \rightarrow 0$. Note that for $\lambda=1.2$ we find that $\alpha_{m^{*}} \rightarrow 0$ and $\alpha_{\Omega_{0}} \rightarrow \infty$ for $\gamma \rightarrow 0$, in agreement with Ref. [12] which predicts two different critical values $\lambda_{c}^{\mathrm{ph}}=1.18$ and $\lambda_{c}^{\mathrm{el}}=1.328$ where a double-well lattice potential and an electronic self-trapping, respectively, occur.

The different physics underlying the regimes sketched by dashed and solid lines is reflected in the overall $\gamma$ dependence of the IEs. In the large coupling regime $\lambda>$ $\lambda_{c}$ the isotope coefficients diverge for $\gamma \rightarrow 0$ and approach monotonically zero in the antiadiabatic limit, in agreement with the Lang-Firsov result. On the other hand, for $\lambda<\lambda_{c}$, an initial increase of the anomalies of $\alpha_{m^{*}}$ and $\alpha_{\Omega_{0}}$ as a function of $\gamma$ is followed by a decrease for $\gamma>\gamma^{*}$, where $\gamma^{*}$ marks the position of the minima and maxima of $\alpha_{m^{*}}$ and $\alpha_{\Omega_{0}}$, respectively.

The values of $\gamma^{*}$ depend strongly on the $e$-ph coupling $\lambda$, and, in principle, a nonadiabatic crossover parameter $\gamma^{*}(\lambda)$ can be defined for both the electronic and phononic properties. According to our previous reasoning and to Eqs. (4) and (5), $\gamma^{*}(\lambda)$ can be interpreted as the curve separating a region in which a perturbative theory based on the adiabatic FL picture can be safely employed $\left(\gamma<\gamma^{*}\right)$ from a region where the Lang-Firsov approach is a more appropriate starting point for a $1 / \gamma$ expansion $\left(\gamma>\gamma^{*}\right)$. In the nonadiabatic crossover $\left(\lambda<\lambda_{c}\right.$ and $\left.\gamma \sim \gamma^{*}\right)$ the anomalies of the IEs are significant $\left(\alpha_{m^{*}} \sim-0.3\right.$ and
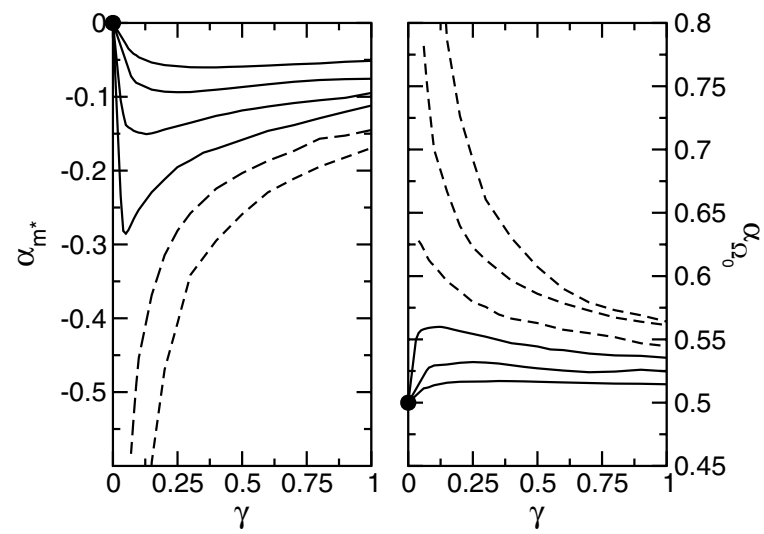

FIG. 3. Dependence of $\alpha_{m^{*}}$ and $\alpha_{\Omega_{0}}$ on the nonadiabatic parameter $\gamma$. Curves are plotted for the $e$-ph coupling $\lambda=$ $0.6,0.8,1.0,1.2,1.4,1.6$ from top to bottom (bottom to top) in left (right) panel. Filled circles mark the adiabatic ME limit. 


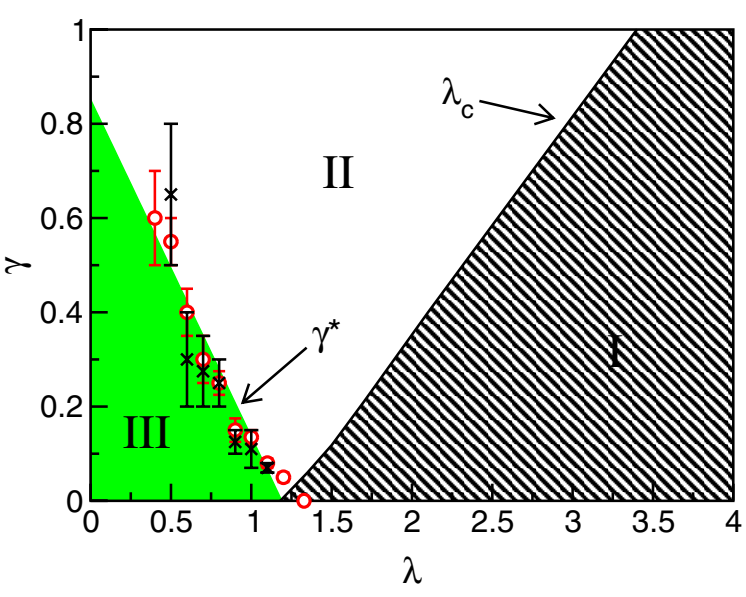

FIG. 4 (color online). Nonadiabatic phase diagram defined by anomalous IEs. Open circles and crosses represent, respectively, the minima and the maxima in the $\gamma$ behavior of $\alpha_{m^{*}}, \alpha_{\Omega_{0}}$. Error bars are not related to the accuracy of the isotope coefficients but rather to the flatness of $\alpha_{m^{*}}, \alpha_{\Omega_{0}}$ as a function of $\gamma$. The curve $\lambda_{c}$ marks the polaron crossover as defined in the text.

$\left.\alpha_{\Omega_{0}} \sim 0.55\right)$ despite such a region being well within the metallic regime and far from the polaronic instability.

The complex phenomenology of the IEs is summarized in Fig. 4, where the nonadiabatic crossover $\gamma^{*}(\lambda)$ is reported for $\alpha_{m^{*}}$ (open circles) and $\alpha_{\Omega_{0}}$ (crosses). We note that $\gamma^{*}$ lies on a universal curve for both the electron and phonon properties, reflecting the fact that electron and lattice degrees of freedom are strictly mixed in the nonadiabatic regime. Such universality is lost for $\gamma \rightarrow 0$, where $\gamma^{*}$ disappears at the two different values $\lambda_{c}^{\mathrm{ph}}$ and $\lambda_{c}^{\mathrm{el}}$ discussed above. Figure 4 also clearly shows that the nonadiabatic crossover $\gamma^{*}(\lambda)$ is not related to the onset of polaronic effects, pinpointed in the figure by the solid line marking the appearance of a bimodal structure in the lattice probability distribution function.

Figure 4 defines three regimes: (I) a polaron region (dashed area) where electrons are almost trapped leading to significant lattice distortions. This effect is strongly affected by lattice quantum fluctuations, triggered by finite $\omega_{0}$, leading to giant IEs. (II) A highly nonadiabatic region (white area) where the system is qualitatively described in terms of itinerant quasiparticle carrying along its hugely fluctuating phonon cloud. In this regime quantum lattice fluctuations relax the mixing between lattice and electronic degrees of freedom leading to a reduction of the anomalous IEs. (III) A weakly nonadiabatic region (gray filled area) where anomalous IEs are tuned by the opening of nonadiabatic channels in the $e$-ph interaction. In this region DMFT qualitatively confirms the results of the nonadia- batic theory described by vertex diagrams in a perturbative approach $[5,6]$.

In conclusion, we have defined a phase diagram of the spinless Holstein model based on the anomalous phenomenology of the IEs on both electronic and phonon properties. In the metallic regime we identified a new nonadiabatic crossover $\gamma^{*}$ (not related to polaronic instability) between different weakly and highly nonadiabatic FL quasiparticle pictures. The largest anomalies in the isotope coefficients are found in this intermediate crossover region, where theoretical and experimental studies suggest $A_{3} \mathrm{C}_{60}$ fullerides [16,17] (see also Fig. 8 of Ref. [18]), cuprates [19], and $\mathrm{MgB}_{2}$ to be located. A rigorous generalization of our results in the presence of electronic correlation and away from the half-filling case is, of course, needed for a quantitative analysis in these complex materials. Preliminary results show that $\alpha_{m^{*}}$ can be significantly suppressed in low filled systems even in a highly nonadiabatic regime, in agreement with recent experimental results on $\mathrm{MgB}_{2}$ [20].

We are grateful to $\mathrm{C}$. Castellani for illuminating discussions. We acknowledge support from Miur Cofin2003 and FIRB RBAU017S8R and INFM PRA-UMBRA.

[1] G. M. Zhao et al., Nature (London) 385, 236 (1997); R. Khasanov et al., Phys. Rev. Lett. 92, 057602 (2004).

[2] D. Rubio Temprano et al., Phys. Rev. Lett. 84, 1990 (2000).

[3] G.-H. Gweon et al., Nature (London) 430, 187 (2004).

[4] G. Zhao et al., Nature (London) 381, 676 (1996).

[5] C. Grimaldi, E. Cappelluti, and L. Pietronero, Europhys. Lett. 42, 667 (1998).

[6] A. Deppeler and A. J. Millis, Phys. Rev. B 65, 224301 (2002).

[7] P. E. Kornilovitch and A. S. Alexandrov, Phys. Rev. B 70, 224511 (2004).

[8] A. Georges et al., Rev. Mod. Phys. 68, 13 (1996).

[9] S. Ciuchi et al., Phys. Rev. B 56, 4494 (1997).

[10] D. Meyer, A. C. Hewson, and R. Bulla, Phys. Rev. Lett. 89, 196401 (2002).

[11] M. Capone and S. Ciuchi, Phys. Rev. Lett. 91, 186405 (2003).

[12] A. J. Millis, R. Mueller, and B. I. Shraiman, Phys. Rev. B 54, 5389 (1996).

[13] J. K. Freericks et al., Phys. Rev. B 48, 6302 (1993).

[14] M. Caffarel and W. Krauth, Phys. Rev. Lett. 72, 1545 (1994).

[15] M. Capone, W. Stephan, and M. Grilli, Phys. Rev. B 56, 4484 (1997); M. Capone, C. Grimaldi, and S. Ciuchi, Europhys. Lett. 42, 523 (1998).

[16] O. Gunnarsson, Rev. Mod. Phys. 69, 575 (1997).

[17] E. Cappelluti et al., Phys. Rev. Lett. 85, 4771 (2000).

[18] E. Cappelluti et al., Eur. Phys. J. B 21, 383 (2001).

[19] M. Kulic, Phys. Rep. 338, 1 (2000).

[20] D. Di Castro et al., Phys. Rev. B 70, 014519 (2004). 